\title{
Physiological remodeling of bifurcation aneurysms: preclinical results of the eCLIPs device
}

\author{
*Thomas R. Marotta, MD, ${ }^{1,2}$ Howard A. Riina, MD, ${ }^{3}$ lan McDougall, BASc, ${ }^{4}$ Donald R. Ricci, MD,, 45 \\ and Monika Killer-Oberpfalzer, MD ${ }^{6}$
}

\begin{abstract}
'Department of Medical Imaging, University of Toronto; 2Diagnostic and Therapeutic Neuroradiology, St. Michael's Hospital, University of Toronto, Ontario; ${ }^{4}$ Evasc Medical Systems Corp., Vancouver; ${ }^{5}$ Division of Cardiology, University of British Columbia, Vancouver, British Columbia, Canada; ${ }^{2}$ Department of Neurosurgery, NYU School of Medicine, NYU Langone Medical Center, New York, New York; and ${ }^{6}$ Department of Neurology/Neuroscience Institute, Christian Doppler Clinic, Paracelsus Medical University, Salzburg, Austria
\end{abstract}

\begin{abstract}
OBJECTIVE Intracranial bifurcation aneurysms are complex lesions for which current therapy, including simple coiling balloon- or stent-assisted coiling, coil retention, or intrasaccular devices, is inadequate. Thromboembolic complications due to a large burden of intraluminal metal, impedance of access to side branches, and a high recurrence rate, due largely to the unmitigated high-pressure flow into the aneurysm (water hammer effect), are among the limitations imposed by current therapy. The authors describe herein a novel device, eCLIPs, and its use in a preclinical laboratory study that suggests the device's design and functional features may overcome many of these limitations.
\end{abstract}

METHODS A preclinical model of wide-necked bifurcation aneurysms in rabbits was used to assess functional features and efficacy of aneurysm occlusion by the eCLIPs device.

RESULTS The eCLIPs device, in bridging the aneurysm neck, allows coil retention, disrupts flow away from the aneurysm, leaves the main vessel and side branches unencumbered by intraluminal metal, and serves as a platform for endothelial growth across the neck, excluding the aneurysm from the circulation.

CONCLUSIONS The eCLIPS device permits physiological remodeling of the bifurcation.

https://thejns.org/doi/abs/10.3171/2016.10.JNS162024

KEY WORDS interventional neuroradiology; animal experiment; intracranial aneurysm; therapeutic embolization; flow diversion; neurosurgery; interventional radiology; bifurcation aneurysm; vascular disorders

$\mathrm{S}$ XTY-FOUR percent of all cerebral aneurysms occur at arterial bifurcations. ${ }^{3}$ To date, there are no commercially available stents and few endoluminal devices that are specifically designed to treat bifurcation aneurysms. Current options for the endovascular treatment of bifurcation aneurysms include 1) use of simple coiling (no stent) and balloon remodeling; 2) use of commercially available stents "off-label" to create a Y- or T-stent in conjunction with coils; 3) placement of coil-retaining devices in the parent artery (e.g., PulseRider [Pulsar Vascular], pCONus Bifurcation Aneurysm Implant [phenox]); and 4) insertion of intrasaccular devices (e.g., Woven EndoBridge [WEB] Aneurysm Embolization System [MicroVentionTerumo], Luna [Medtronic]). All of these techniques have limitations, particularly an important rate of aneurysm recurrence. A key factor responsible for recurrence at a bifurcation is speculated to be the water hammer effect (flow jet phenomenon) of blood under pressure from the main vessel, unimpeded at the neck, directly into the aneurysm..$^{5,6,15}$ Thus, a therapy specifically designed to treat these complex lesions is needed.

In this paper, we describe our preclinical experience with the eCLIPs device (Evasc Medical Systems Corp.), a novel endovascular device designed specifically for treating intracranial aneurysms arising at bifurcations. We describe the device's design and functional features, including its coil retention and flow-disrupting characteristics.

\section{Methods \\ Device Description and Technology}

The eCLIPs Bifurcation Remodelling System (eBRS)

ABBREVIATIONS eBRS $=$ eCLIPs Bifurcation Remodelling System

SUBMITTED August 3, 2016. ACCEPTED October 31, 2016.

INCLUDE WHEN CITING Published online March 17, 2017; DOI: 10.3171/2016.10.JNS162024

* Drs. Marotta, Riina, and Ricci and Mr. McDougall contributed equally to this work. 


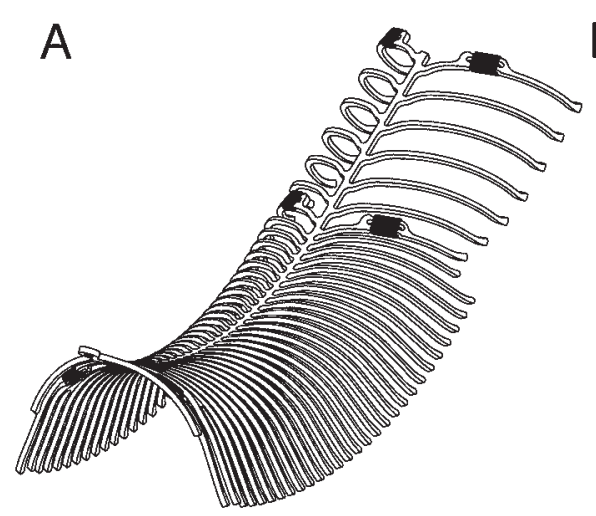

FIG. 1. eCLIPs device: 3D view (A), side view (B), plan view (C).

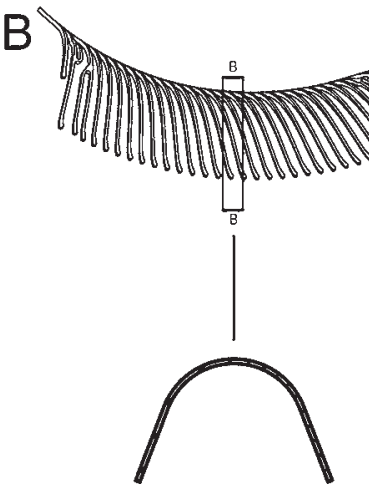

Cross-section B-B

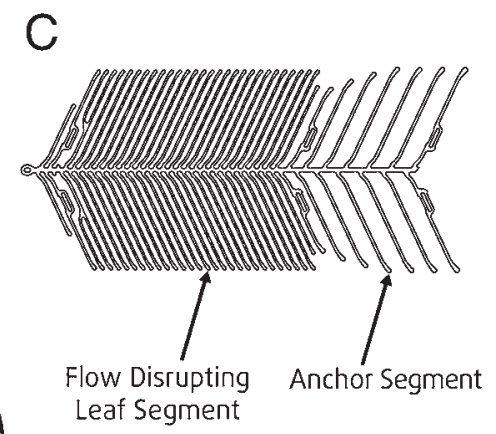

Cross-section A-A

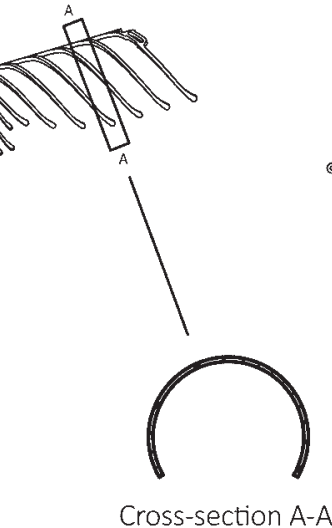

A comprises 4 components: the implantable device and 3 components used in the delivery of the device (microcatheter, microintroducer, and hypotube mechanical detachment system). The eCLIPs device is the implanted device portion of the eBRS and is made of a noncircumferential nitinol frame with 6 radiopaque marker bands (Fig. 1).

Compared with the Y- or T-stenting technique, there is less metal in the branch arteries and no metal in the parent artery. The eCLIPs device has a "spine-rib" design with 2 types of ribs emanating from the spine, the first of which is a low-density anchor segment that secures the device in one branch vessel with ribs of a semirounded splay.

The comparative resistive force of this anchor segment, illustrated in Table $1,{ }^{14}$ is greater than commonly available tubular stents, but less than tubular flow diverters such as Pipeline.

The second type of rib (Fig. 1B), broadly splayed, comprises a high-density leaf segment that bridges the aneurysm neck. The spine itself prevents shortening on deployment and the curved nature of the spine serves a self-orienting function across the neck. The ribs of the leaf segment 1) allow passage of a microcatheter to enable the placement and retention of embolic coils within the aneurysm sac, 2) provide for a flow diversion effect to mitigate the water hammer or jet effect of flow into the aneurysm from the main vessel, and 3) serve as a platform for endothelial growth. The noncircumferential design improves wall apposition over tubular structures and allows preservation of flow into adjacent collaterals and ready catheter access to branch vessels.

\section{Method of Deployment and Confirmation of Positional Precision of eCLIPs at a Bifurcation Aneurysm}

Standard coaxial techniques are used, first to engage a microcatheter into a side branch, then to advance the eCLIPs device to the tip of the microcatheter (Fig. 2A, a), then to withdraw the microcatheter to expose first the anchor segment, then the entire device (Fig. 2A, b). The guidewire is then pulled back from the first side branch and advanced into the other side branch (Fig. 2A, c), and the device is railed over the wire to lie across the neck and into the second branch so that the leaf segment bridges the neck (Fig. 2A, d). At any time up to and including this step, the device can be pulled back into the microcatheter, repositioned, or removed. When the device is positionally acceptable, it is detached from the delivery hypotube, and then coils can be inserted into the aneurysm in the usual fashion (Fig. 2A, e and f).

Leaf orientation across the neck (Fig. 2B), ensuring that the leaf adequately covers the neck, is determined by the relative position of the paired markers. If the markers are overlapped (or nearly so), the device is appropriately oriented across the neck.

There is considerable latitude in overlap of the markers because they are placed close to the spine and the leaf has a coverage of $210^{\circ}-266^{\circ}$ when placed in a $2-$ to $3.25-\mathrm{mm}$ diameter vessel; depending on the breadth of the neck (not width), the spine can be $0^{\circ}-45^{\circ}$ out of orientation and the neck will still be covered by the broadly splayed ribs of the leaf section. Figure $2 \mathrm{C}$ illustrates, in a lateral view, the "breadth" of the aneurysm with the markers depicting that the spine is placed right beneath the neck; it also shows how the "length" of the ribs will cover a broad neck even if the spine is not exactly beneath the middle of the neck.

\section{In Vivo Model to Test eCLIPs Proof of Principle}

We assessed the utility of eCLIPs in a rabbit model for bifurcation aneurysms prepared by Monika Killer-Oberpfalzer's team at Universitätsklinikum Christian Doppler Klinik, Paracelsus Medical University, Salzburg., ${ }^{1,8}$ The Animal Care and Use Committee at this institution approved the animal experimentation in this study. This

TABLE 1. Comparative resistive force of the eCLIPs anchor segment versus stents

\begin{tabular}{lc}
\hline \multicolumn{1}{c}{ Device (manufacturer) } & $\begin{array}{c}\text { Radial Force at } 50 \% \text { Compression } \\
\text { (flat plate test) in N/mm }\end{array}$ \\
\hline Leo (Balt) & 0.0037 \\
\hline Neuroform3 (Stryker) & 0.0065 \\
\hline Enterprise (Codman) & 0.0082 \\
\hline Solitaire (Medtronic) & 0.0106 \\
\hline Wingspan (Stryker) & 0.0116 \\
\hline eCLIPs & 0.0238 \\
\hline Pipeline $5 \times 12$ (Medtronic) & 0.0981 \\
\hline Pipeline $4 \times 25$ & 0.1138 \\
\hline
\end{tabular}




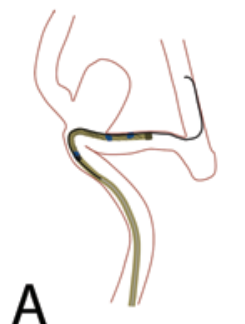

a

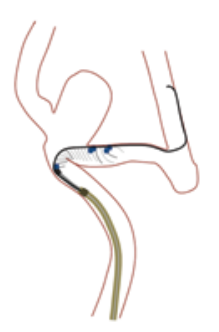

b

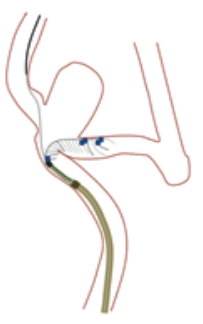

C

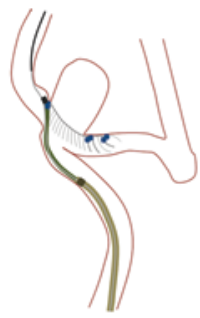

d

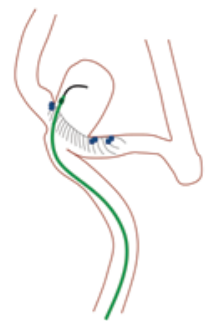

e

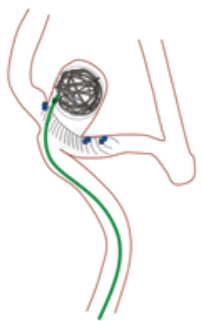

$f$

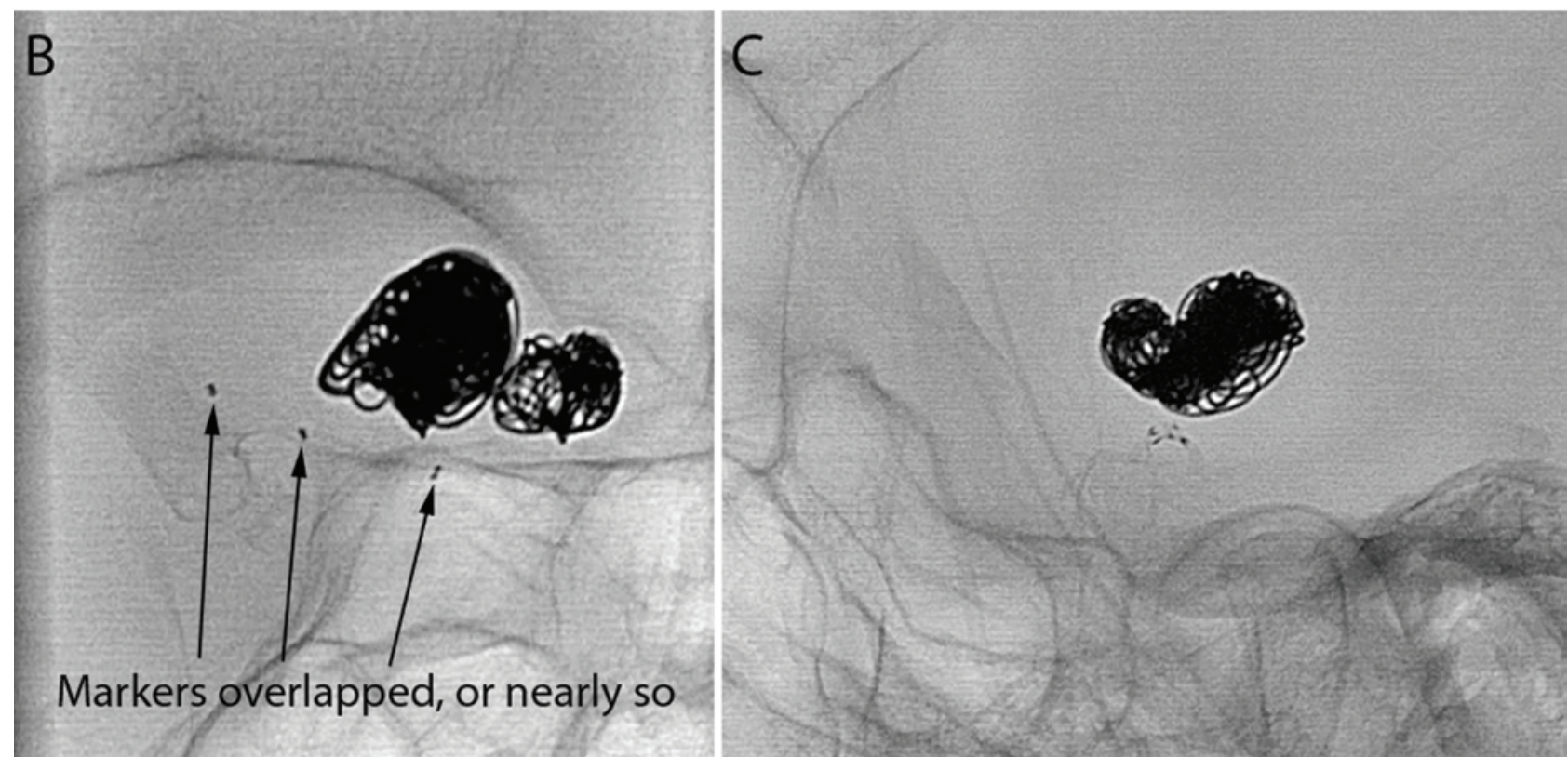

FIG. 2. A: Illustrated eCLIPs deployment sequence. B and C: Radiographic images of a clinical implant. Frontal view of implanted eCLIPs, with overlapped markers, showing appropriate orientation of the leaf segment across the neck, delimited by the middle and rightmost set of markers (arrows, B). Lateral view showing 3 pairs of markers immediately beneath the neck (C).

model has been used extensively to assess the utility of devices for management of bifurcation aneurysms. ${ }^{13}$ The animals underwent pretreatment with aspirin and clopidogrel; both were continued through 30 days. An eCLIPs device was implanted at the site of the bifurcation aneurysm in each of 6 rabbits (Evasc Medical Systems Corp., Animal Study Results Study 110-1201, 2013). Functionality of all components of the eBRS system was assessed during these implants. During the implantation procedure, before detachment, the device was unsheathed and resheathed several times to confirm this recapture functionality. The paired radiopaque markers satisfactorily identified appropriate device orientation. Immediately after detachment, angiography was repeated to determine the degree of stasis within the aneurysm, a surrogate for intrinsic flow diversion by the high-density leaf segment. In 5 animals, the aneurysm was coiled immediately after device deployment, with the device's leaf segment being crossed several times to confirm stability of the implanted device. In 1 animal the aneurysm was coiled at 30 days to confirm that the leaf segment could be crossed and the aneurysm could be accessed significantly later after the index procedure, if necessary. Four animals underwent additional angiography at 30 days, followed by euthanization, and 2 animals underwent angiography at 30 days and again at 90 days, followed by euthanization. On each occasion of follow-up angiography, the side branches adjacent to the aneurysm were accessed by the guidewire to confirm unimpeded access.

\section{Results}

A summary of results is shown in Table 2. There was no evidence of migration of the device at any time point during or after implantation, and the main branch and all side branches remained fully patent, with no evidence of vessel stenosis. Thirty-day angiography showed Raymond-Roy Class 1 or 2 aneurysm closure ${ }^{19}$ in 4 of 5 aneurysms subjected to coiling at the index procedure, and the other showed Raymond-Roy Class 3 closure. The uncoiled aneurysm showed Raymond-Roy Class 3 at 30 days, but it still had flow stasis and underwent coiling at this stage with the 90-day angiography showing Raymond-Roy Class 1. The other aneurysm with index loose coiling showed progressive occlusion through the 30- and 90-day angiography. High-resolution radiography performed during histological preparation disclosed fractures in 5 of 6 devices, all in the spine near the transition of the anchor to the leaf segment. Subsequent evaluation showed no evidence of corrosion at the sites. Repeated deployment and 
TABLE 2. Summary of results

\begin{tabular}{lccc}
\hline \multicolumn{1}{c}{ Result } & Procedure & 30 Days & 90 Days \\
\hline Device migration & None & None & None \\
\hline Branch patency & Complete & Complete & Complete \\
\hline Vessel dissection/stenosis & None & None & None \\
\hline Coiled & 5 & 1 & \\
\hline Angiography & 6 & 6 & 2 \\
\hline Stasis & 6 & & \\
\hline Raymond Class 1 & & 2 & 1 \\
\hline Raymond Class 2 & & 3 & 1 \\
\hline Raymond Class 3 & & 1 & \\
\hline
\end{tabular}

retraction in an access catheter to test durability on the bench failed to reproduce fractures. In in vitro-simulated use, fatigue testing (400 million cycles, approximating 10 years) also failed to reproduce fractures (eVasc Medical Systems, Corp., RPT-0267 eCLIPs Bifurcation Device Durability Study 110-1301 Test Report Rev A, 2013). Finally, analysis of the rabbit's neck movements showed that the tissue surrounding the aneurysm is highly mobile, resulting in repeated bending and straightening of the implanted device at the site of fracture with swallowing and moving the head side to side, and compression of the device with scratching of the neck. Thus, we concluded that fractures occurred as a result of nonphysiological move- ment of the device unique to the animal model that would not be recreated in a clinical scenario, and that the animal model used herein is not designed to assess durability of the device.

\section{Angiographic and Histopathological Examples}

A representative series of angiograms, from preimplantation through 1 month of follow-up, is shown in Fig. 3A. The aneurysm is loosely coiled, with Raymond-Roy Class 3 postimplant and coiling. At the 30-day follow-up, the aneurysm had a Raymond-Roy Class 1 closure status (completely excluded from the circulation). The markers are evident, particularly in the right-most frame, with no contrast in the vessel. There is no evidence of device migration, and the main and side branches are fully patent, with no stenosis.

Histologically (Fig. 3B-D), it is evident that the eCLIPs device ribs are completely incorporated into the vessel wall, with organized smooth muscle and neointimal growth over the aneurysm neck. This degree of healing supports the notion that the device was well apposed to the vessel wall on implantation. There was no evidence of an inflammatory response. The endothelial growth across the entire neck of the aneurysm, excluding it from the circulation, allows for complete cicatrization of the aneurysm itself.

Another representative series of angiograms, from preimplantation to the 90-day follow-up, is shown in Fig. 4A.
A

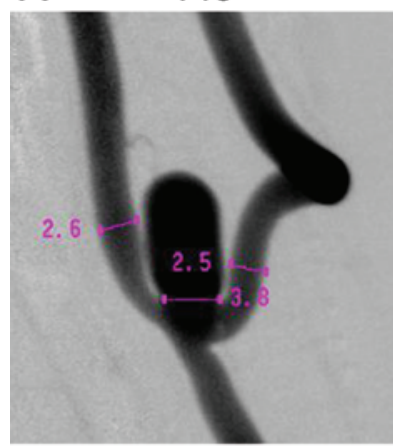

Post Implant

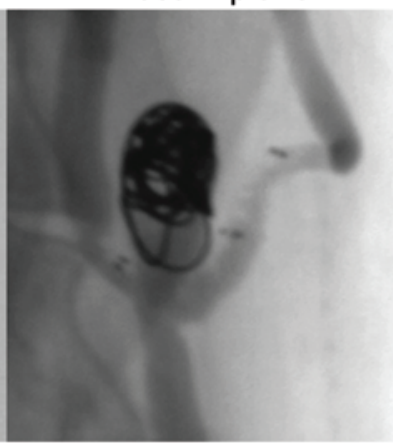

30-Day Follow-Up
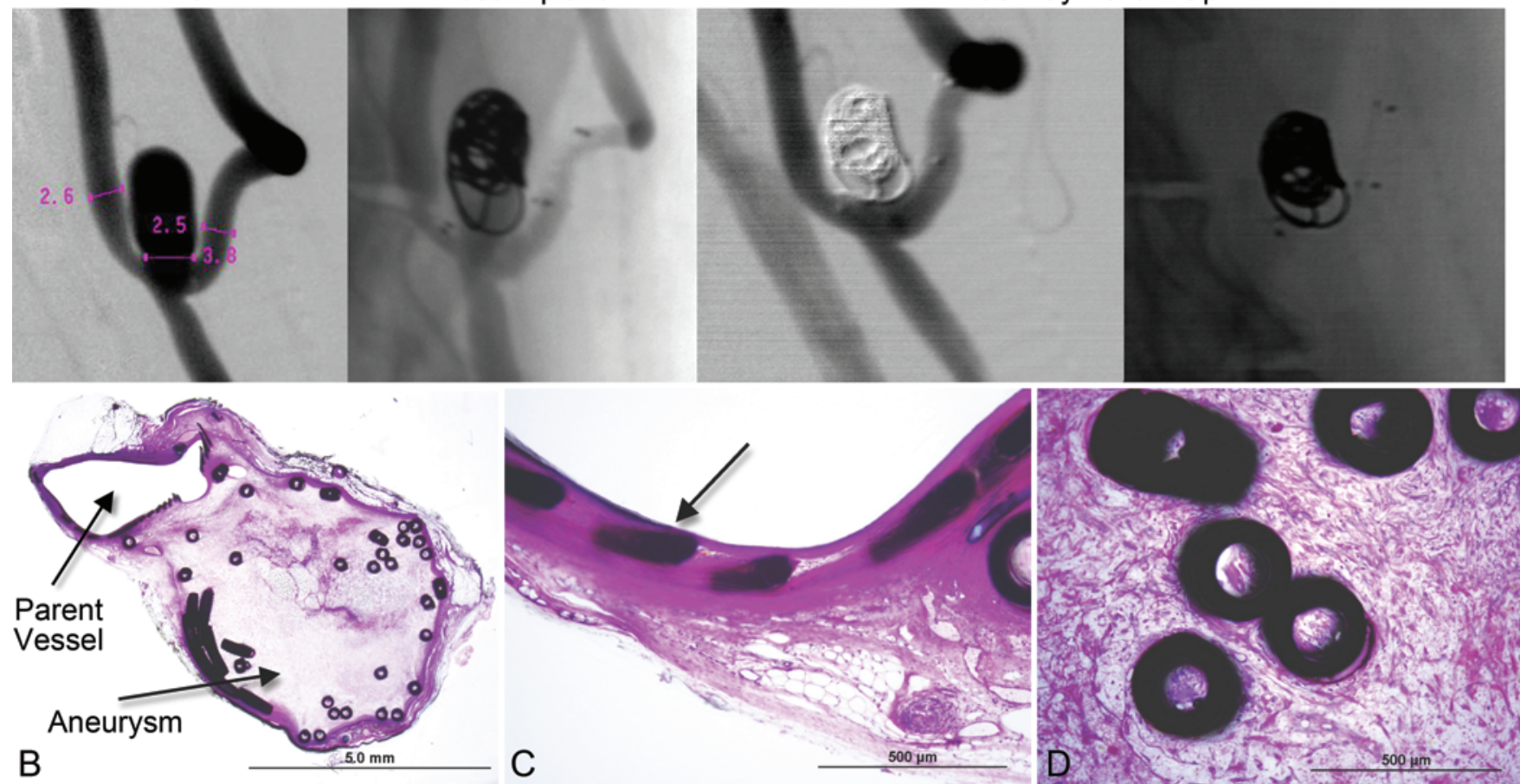

C

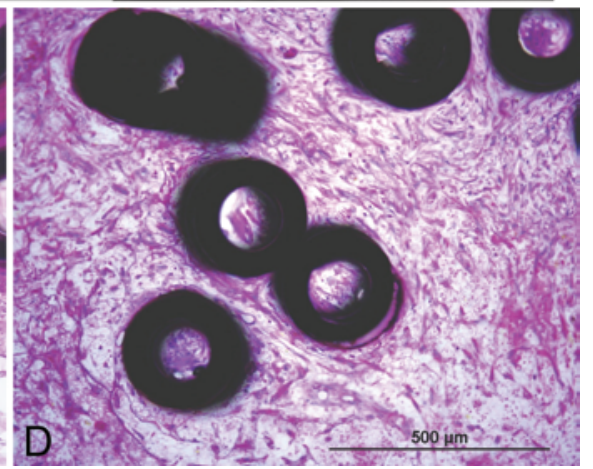

FIG. 3. A: Serial angiograms obtained preimplantation to 30 days postimplantation, showing a bifurcation aneurysm in a rabbit treated with eCLIPS. B-D: Histological images showing a cross-section of the parent lumen in the area of the aneurysm sac (B) 30 days after eCLIPs implantation and loose coiling. The lumen is widely patent, and the eCLIPs device ribs are completely incorporated with organized smooth muscle neointimal growth over the aneurysm neck. Black circles within the aneurysm represent coils. Close-up view of the parent vessel wall with neointimal overgrowth incorporating a device rib (arrow, C). Close-up view of the aneurysm sac (D), showing embolic coils incorporated by the fibrous matrix of the aneurysm sac. Toluidine blue/basic fuchsin stain. Courtesy Renu Virmani, CVPath Institute, Inc., Gaithersburg, MD. 

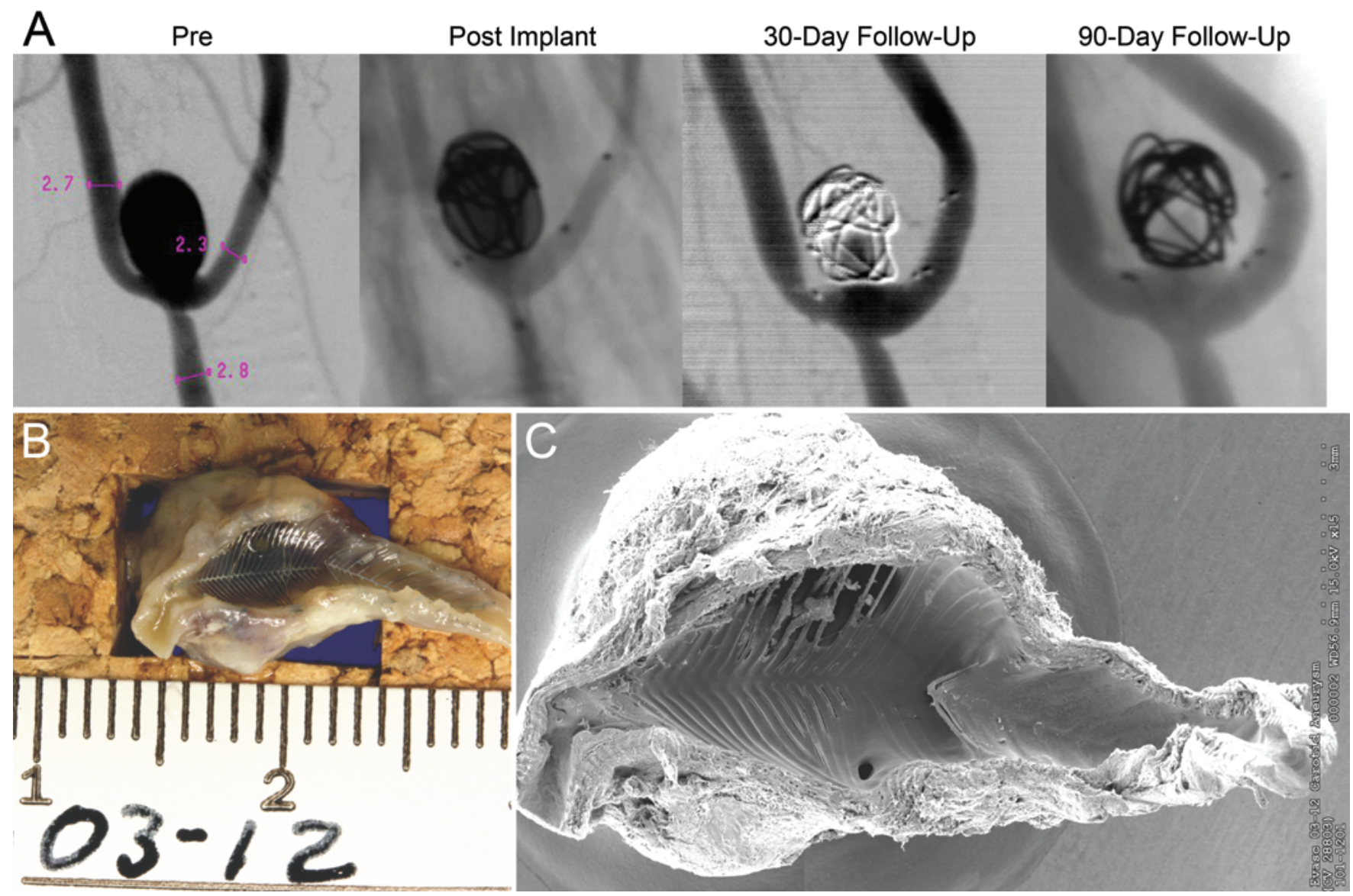

FIG. 4. A: Serial angiograms obtained preimplantation to 90 days postimplantation, showing a bifurcation aneurysm in a rabbit treated with eCLIPs. B and C: Gross pathology (B) and scanning electron microscopy (C, original magnification $\times 15)$ of eCLIPs and aneurysm explanted from the animal at 90 days. Courtesy Renu Virmani, CVPath Institute, Inc., Gaithersburg, MD.

The aneurysm is loosely coiled, with some stasis within the aneurysm evident after implantation and coiling. At 30 and 90 days there is progressive thrombosis within the aneurysm space. The markers are clearly evident. There is no evidence of device migration, and the main and side branches are fully patent, with no stenosis.

Gross pathology and scanning electron microscopy of the explanted device and aneurysm (Fig. 4B and C) show that the device, both the leaf (to the left in the images) and anchor segments (to the right), is largely incorporated into the vessel wall and is endothelialized across the neck of the aneurysm that is "behind" the plane of the spine and ribs as seen in the images. There is no evidence of platelet accumulation or adhesion to the endothelium, or any thrombus formation. Endothelium missing from the leaf segment ribs is either a region with incomplete incorporation of the ribs, or an artifact of the preparation technique. The fracture of the spine at the transition of the leaf and anchor segments is evident.

Another representative series of angiograms, from preimplantation to the 30-day follow-up, is shown in Fig. 5A. The aneurysm is densely packed, and shows RaymondRoy Class 2 occlusion immediately after implantation and Raymond-Roy Class 1 occlusion at the 30-day follow-up.

Scanning electron microscopy (Fig. 5B) shows endothelialization of the device and exclusion of the aneurysm.
The ribs are covered by endothelium, but they are not yet fully incorporated in the wall. There is no platelet accumulation or thrombus formation.

\section{Discussion}

This self-expanding nitinol eCLIPs device shows similar functional characteristics to its first-generation conceptual predecessor, a balloon-expandable stainless steel tubular device with a polymer covering over an independently moveable leaf section that, at 30 days postimplantation in a swine model, completely isolated the aneurysm from the circulation. ${ }^{16}$

The eCLIPs device was designed specifically to address several predetermined features believed to be required to treat bifurcation aneurysms by endovascular means: removable device, retractable and repositionable before detachment, nonshortening on deployment, absence of device migration, stable platform during coil delivery, coil retention, flow disruption away from the aneurysm, platform for endothelial growth, no compromise of access to side branches, and good wall apposition. Even aneurysms coiled loosely resulted in complete occlusion (RaymondRoy Class 1), likely due to the combination of flow disruption and the growth of endothelial tissue over the leaf segment. This animal study demonstrates that the eCLIPs 


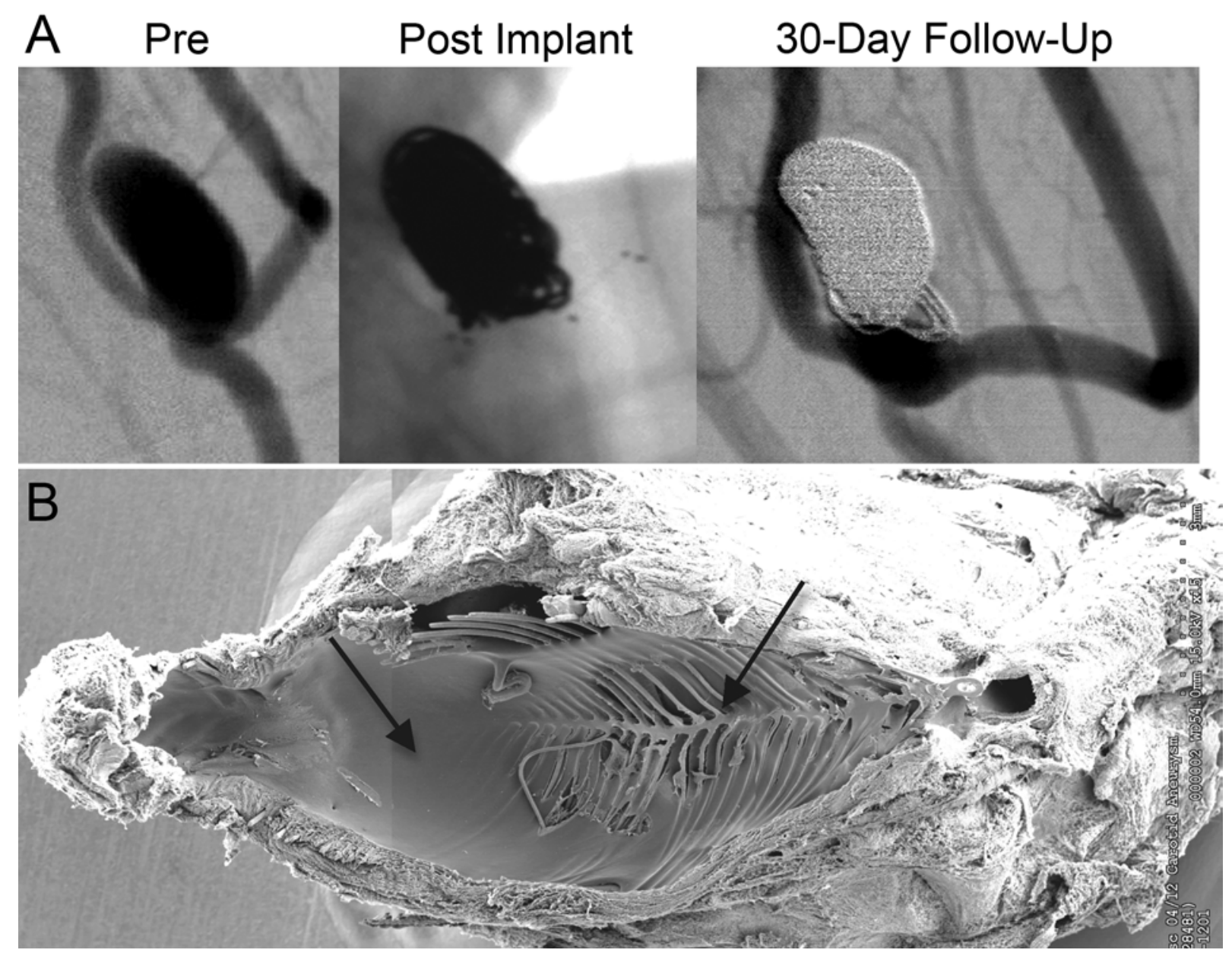

FIG. 5. A: Serial angiograms, from preimplantation to 30 days postimplantation, of a bifurcation aneurysm in rabbit treated with eCLIPs. B: Scanning electron microscopy of the eCLIPs device and aneurysm explanted from the animal model at 30 days (original magnification $\times 15$ ) shows endothelialization of the device and exclusion of the aneurysm (left arrow). The ribs are covered by endothelium, but they are not yet fully incorporated in the wall (right arrow). Courtesy Renu Virmani, CVPath Institute, Inc., Gaithersburg, MD.

device satisfactorily addresses these features. As such, it facilitates physiological remodeling of the aneurysm neck so that, 30-90 days after implantation in a rabbit model, the device is incorporated into the vessel to completely exclude the aneurysm from the circulation and is effectively thereafter "invisible" to the circulation.

These results suggest that there is promise in the clinical arena for a much-needed reduced recurrence rate after initial treatment of bifurcation aneurysms. Other approaches to management of bifurcation aneurysms that are not amenable to "simple" coiling such as those with wide necks and a low dome/neck ratio, include stent-assisted coiling, other device-assisted coiling (e.g., pCONus and PulseRider), and intrasaccular devices (e.g., Luna and WEB). None of these devices is designed to bridge the neck to disrupt blood flow and mitigate the water hammer effect that is a major factor in aneurysm persistence or recurrence after coiling through the mechanism of compaction of coils and compression of intrasaccular devices. And none serves as a platform for endothelial growth across the neck, the ultimate factor in preventing aneurysm recurrence.

Simple coiling has a high aneurysm recurrence rate for bifurcation aneurysms compared with sidewall aneurysms (35\% at an average follow-up of 30 months $^{12}$ ), to a large degree due to coil compaction. ${ }^{10}$ Stent-assisted coiling using various stents and various techniques has not improved on the aneurysm recurrence rate at between $18 \%$ and $37 \%$., 2,4,11,18,20

Newer coil retention devices such as pCONus and PulseRider are placed from the main trunk (parent vessel) at the neck of the aneurysm with the sole goal of preventing coils from embolizing or extruding from the aneurysm into the main or branch (daughter) vessels. By the nature of their design, access to branch vessels may be impeded and lack of wall apposition in the main branch may promote thrombus development. No comprehensive followup data are available for pCONus, while PulseRider has a consistent 20\% Raymond-Roy Class 3 occlusion score at follow-up. ${ }^{7,9}$

Intrasaccular devices (e.g., Luna and WEB) have the goal of occupying much of the aneurysm space, preventing free flow of blood within the aneurysm sac and thereby encouraging thrombotic obliteration. Challenges include matching the device to the size and morphology of the an- 
eurysm, and complications related to a relatively solid and fixed intrasaccular device exposed to the jet effect of blood from the main vessel at the neck. The most recent data from a combined WEB trial show an $18 \%$ recurrence rate at a mean follow-up of 5.3 months, ${ }^{17}$ though a recent report shows a significant incidence of WEB compression with a consequent recurrence rate up to $36 \%$. $^{5}$

Whether the eCLIPs device, with all its favorable design elements, will reduce the incidence of aneurysm recurrence remains to be determined from clinical trials.

\section{Limitations of the Study}

Limitations of the study include a small sample size, and lack of independent analysis of angiographic results and longer-term results.

\section{Conclusions}

Preclinical assessment of eCLIPs device in a rabbit model of bifurcation aneurysms shows adherence to several features required for definitive and sustained aneurysm exclusion from the circulation and incorporation of the device into the vessel wall to produce a physiological remodeling of the aneurysm. Clinical studies are required to determine if these favorable features are translatable to the clinical arena.

\section{References}

1. Bavinzski G, al-Schameri A, Killer M, Schwendenwein I, Gruber A, Saringer W, et al: Experimental bifurcation aneurysm: a model for in vivo evaluation of endovascular techniques. Minim Invasive Neurosurg 41:129-132, 1998

2. Biondi A, Janardhan V, Katz JM, Salvaggio K, Riina HA, Gobin YP: Neuroform stent-assisted coil embolization of wide-neck intracranial aneurysms: strategies in stent deployment and midterm follow-up. Neurosurgery 61:460-469, 2007

3. Brisman JL, Song JK, Newell DW: Cerebral aneurysms. N Engl J Med 355:928-939, 2006

4. Chalouhi N, Jabbour P, Gonzalez LF, Dumont AS, Rosenwasser R, Starke RM, et al: Safety and efficacy of endovascular treatment of basilar tip aneurysms by coiling with and without stent assistance: a review of 235 cases. Neurosurgery 71:785-794, 2012

5. Cognard C, Januel AC: Remnants and recurrences after the use of the WEB intrasaccular device in large-neck bifurcation aneurysms. Neurosurgery 76:522-530, 2015

6. Damsa T, Appel E, Cristidis V: "Blood-hammer" phenomenon in cerebral hemodynamics. Math Biosci 29:193-202, 1976

7. Fischer S, Weber A, Titschert A, Brenke C, Kowoll A, Weber W: Single-center experience in the endovascular treatment of wide-necked intracranial aneurysms with a bridging intra-/ extra-aneurysm implant (pCONus). J Neurointerv Surg 8:1186-1191, 2016

8. Forrest MD, O'Reilly GV: Production of experimental aneurysms at a surgically created arterial bifurcation. AJNR Am J Neuroradiol 10:400-402, 1989

9. Gory B, Aguilar-Pérez M, Pomero E, Turjman F, Weber W, Fischer S, et al: pCONus device for the endovascular treatment of wide-neck middle cerebral artery aneurysms. AJNR Am J Neuroradiol 36:1735-1740, 2015

10. Henkes H, Fischer S, Mariushi W, Weber W, Liebig T, Miloslavski E, et al: Angiographic and clinical results in 316 coil- treated basilar artery bifurcation aneurysms. J Neurosurg 103:990-999, 2005

11. Hetts SW, Turk A, English JD, Dowd CF, Mocco J, Prestigiacomo C, et al: Stent-assisted coiling versus coiling alone in unruptured intracranial aneurysms in the matrix and platinum science trial: safety, efficacy, and mid-term outcomes. AJNR Am J Neuroradiol 35:698-705, 2014

12. Jin SC, Kwon OK, Oh CW, Bang JS, Hwang G, Park NM, et al: Simple coiling using single or multiple catheters without balloons or stents in middle cerebral artery bifurcation aneurysms. Neuroradiology 55:321-326, 2013

13. Killer M, Kallmes DF, McCoy MR, Ding YH, Shum JC, Cruise GM: Angiographic and histologic comparison of experimental aneurysms embolized with hydrogel filaments. AJNR Am J Neuroradiol 30:1488-1495, 2009

14. Krischek O, Miloslavski E, Fischer S, Shrivastava S, Henkes $\mathrm{H}$ : A comparison of functional and physical properties of self-expanding intracranial stents [Neuroform3, Wingspan, Solitaire, Leo+, Enterprise]. Minim Invasive Neurosurg 54:21-28, 2011

15. Kwan ESK, Heilman CB, Shucart WA, Klucznik RP: Enlargement of basilar artery aneurysms following balloon occlusion - "water-hammer effect". Report of two cases. J Neurosurg 75:963-968, 1991

16. Marotta TR, Gunnarsson T, Penn I, Ricci DR, McDougall I, Marko A, et al: A novel endovascular clip system for the treatment of intracranial aneurysms: technology, concept, and initial experimental results. Laboratory investigation. J Neurosurg 108:1230-1240, 2008

17. Pierot L, Spelle L, Molyneux A, Byrne J: Clinical and anatomical follow-up in patients with aneurysms treated with the WEB device: 1-year follow-up report in the cumulated population of 2 prospective, multicenter series (WEBCAST and French Observatory). Neurosurgery 78:133-141, 2016

18. Piotin M, Blanc R: Balloons and stents in the endovascular treatment of cerebral aneurysms: vascular anatomy remodeled. Front Neurol 5:41, 2014

19. Roy D, Milot G, Raymond J: Endovascular treatment of unruptured aneurysms. Stroke 32:1998-2004, 2001

20. Weber W, Bendszus M, Kis B, Boulanger T, Solymosi L, Kühne D: A new self-expanding nitinol stent (Enterprise) for the treatment of wide-necked intracranial aneurysms: initial clinical and angiographic results in 31 aneurysms. Neuroradiology 49:555-561, 2007

\section{Disclosures}

The preclinical trial was supported entirely by Evasc Medical Systems Corp., Vancouver, BC, Canada. The authors also report the following. Dr. Marotta: financial stakeholder in Evasc Neurovascular, and medical consultant and proctor for eCLIPs, part of Evasc. Dr. Riina: financial stakeholder in Evasc Neurovascular. Mr. McDougall: employee of Evasc Medical Systems Corp. Dr. Ricci: financial stakeholder in Evasc Neurovascular.

\section{Author Contributions}

Conception and design: Ricci, Killer-Oberpfalzer. Acquisition of data: McDougall, Ricci, Killer-Oberpfalzer. Analysis and interpretation of data: Marotta, Riina, Ricci. Drafting the article: Ricci. Critically revising the article: all authors. Reviewed submitted version of manuscript: all authors. Administrative/technical/ material support: Ricci. Study supervision: Ricci.

\section{Correspondence}

Thomas R. Marotta, Diagnostic and Therapeutic Neuroradiology, St. Michael's Hospital, 30 Bond St., Toronto, ON M5B 1W8, Canada.email: marottat@smh.ca. 